\title{
Neon adsorbed in carbon nanotube bundles
}

\author{
M. C. Gordillo \\ Departamento de Ciencias Ambientales, Facultad de Ciencias Experimentales, Universidad Pablo de Olavide, Carretera de Utrera km 1, \\ 41013, Sevilla, Spain \\ L. Brualla and S. Fantoni \\ International School for Advanced Studies SISSA and INFM DEMOCRITOS National Simulation Center, Via Beirut 2-4, I-34014, \\ Trieste, Italy \\ (Received 17 May 2004; published 17 December 2004)
}

\begin{abstract}
By means of path integral Monte Carlo calculations we were able to estimate the chemical potential of $\mathrm{Ne}$ atoms adsorbed in the interstitial channels of a bundle of $(10,10)$ carbon nanotubes. This allows us to estimate the temperature and pressure conditions for Ne adsorption in those channels. We also calculate the conditions for Ne filling of a bundle of $(5,5)$ tubes with their caps removed.
\end{abstract}

DOI: $10.1103 /$ PhysRevB.70.245420

PACS number(s): 68.90.+g, 05.30.-d

\section{INTRODUCTION}

A bundle of carbon nanotubes (CN's) is a stack of these long and narrow tubes arranged parallel, and as close as possible, to each other. If they are narrow enough, they are basically perfect cylinders ${ }^{1}$ and the entire system has a transversal hexagonal section. Usually, CN's are closed capped structures, meaning that the only possible way for an external species to adsorb is to enter the triangularlike interchannels between every three cylinders or to stay in the valleys formed between every two of them in the outer surface of the bundle, usually termed grooves. However, those interstitial channels are exceedingly narrow, with radius not larger than approximately $3 \AA$. Such a small cross section severely limits the range of adequate adsorbates to small atoms or molecules, such as $\mathrm{He}, \mathrm{H}_{2}$, or $\mathrm{Ne} . \mathrm{H}_{2}$ is a case of especial interest, ${ }^{2}$ since it has been proposed that the hydrogen intake of $\mathrm{CN}$ bundles could be enough to use them as fuel cells for electric vehicles. Unfortunately, this purported capability is somewhat controversial. ${ }^{3-5}$ In any case, the storage capacity of the bundle may increase if the caps of the tubes are removed, hence allowing atoms to enter inside the tubes themselves.

In this work we consider the case of neon adsorbed in a $\mathrm{CN}$ bundle. In particular, we study the adsorption inside two different kinds of channels: the interstices of a $(10,10) \mathrm{CN}$ bundle (with the complementary possibility of Ne adsorption on the grooves); and inside the tubes themselves, using a bundle of $(5,5)$ CN's. Obviously, to consider this last possibility one would have to remove the cylinder caps. Up until now, there has been much theoretical (Ref. 6 and references therein) and experimental work on the adsorption of gases $^{7-10}$ inside and on the outer surface of $\mathrm{CN}$ bundles. This paper together with Ref. 11 provide full quantum many-body finite-temperature results on this issue, and the influence of entropy on quasi-one-dimensional adsorption is taken into account via a chemical potential calculation. This would allow us to predict under what conditions of pressure and temperature adsorption is produced.

The importance of neon adsorption in CN's is twofold. First of all, there is an apparent contradiction between the experimental results, which show that adsorption inside the interstices is nonexistent, and what one would expect. On the basis of the size of neon and the $\mathrm{CN}$ channels, the former clearly fits into $(10,10)$ interstices or $(5,5)$ nanotubes. Furthermore, the binding energy between a $\mathrm{Ne}$ atom inside an interchannel and the surrounding CN's is of the order of $1000 \mathrm{~K},{ }^{11,12}$ and therefore adsorption should take place. Despite these facts neon adsorption has not been observed. ${ }^{8}$ Second, a better understanding of neon adsorbed in CN's will certainly help in solving the puzzle around the hydrogen intake capacity of $\mathrm{CN}$ bundles. Many of the conclusions obtained for neon can be safely assumed for hydrogen, based on the fact that the sizes of $\mathrm{Ne}$ and $\mathrm{H}_{2}$ are similar, and the interatomic potentials resemble each other.

In this paper, we used path integral Monte Carlo (PIMC) calculations to study the adsorption of $\mathrm{Ne}$ atoms in the interchannels between tubes and on the outer valleys between two cylinders. From the energy, which is the primary output of any PIMC calculation, we were able to obtain the chemical potential of $\mathrm{Ne}$ in the interstices and grooves using standard thermodynamic relations. The results obtained indicate that, at some conditions of temperature and pressure given in Ref. 8 for $(10,10)$ capped tubes, the interstices seem to be filled, while the grooves are still clean. The plan of the article is as follows. In Sec. II, we describe how to obtain the chemical potential from the energy per atom. We also transform, by means of the binding energies of a single $\mathrm{Ne}$ atom, the onedimensional (1D) chemical potential into the chemical potential of neon in the interstices of the bundle. The results will be discussed in Sec. III, where the differences between our results and the extant experimental data are discussed. To complete the study, and with identical techniques, we will consider the case of a bundle of $(5,5)$ open carbon nanotubes. There, $\mathrm{Ne}$ atoms should go inside the tubes themselves, due to the very small size of the interchannels between three tubes.

\section{METHOD}

Due to their narrowness, neon gas is almost localized inside the interstitial channels of a $(10,10) \mathrm{CN}$ bundle; there- 
fore, it can be safely described by a one-dimensional model. The underlying assumption of the 1D model is that the effect of the nanotubes surrounding neon is mostly that of localization. Therefore, in our calculation we compute the energy per particle of a $1 \mathrm{D}{ }^{20} \mathrm{Ne}$, by using a model Hamiltonian that includes only the interaction between the Ne atoms. The effect of the surrounding tubes is then added perturbatively. The energy per particle is calculated at different temperatures, namely, 20, 30, 40, and $50 \mathrm{~K}$, and for different densities, by using a PIMC method. Standard periodic boundary 1D conditions were used.

The validity of the one-dimensional model was checked in a similar situation for $\mathrm{H}_{2},{ }^{13,14}$ and proved to be an excellent approximation. The small radius of a $(10,10)$ interstice, or a $(5,5)$ tube, guarantees that a similar treatment would be also correct.

The PIMC method allows the calculation of thermodynamical properties of quantum systems at finite temperatures by mapping each atom into a classical closed polymer ring formed by a variable number of beads connected to each other by springs. ${ }^{15}$ Beads belonging to different atoms interact with each other by a fraction of the real $\mathrm{Ne}-\mathrm{Ne}$ potential. ${ }^{16} \mathrm{We}$ did not consider the possibility of exchange between atoms, since for temperatures higher than $10 \mathrm{~K}$ quantum effects due to indistinguishability of particles can be neglected. This even holds for $\mathrm{H}_{2}$, a species ten times lighter than Ne. ${ }^{17}$

In addition to the $\mathrm{Ne}-\mathrm{Ne}$ interaction inside one channel, we have also considered the binding energy between these $1 \mathrm{D}$ atoms and the three (two) carbon nanotubes around them, in the case of the $(10,10)$ interstice (groove). Those energies have been obtained by diffusion Monte Carlo (DMC) calculations of the energy of a single ${ }^{20} \mathrm{Ne}$ atom inside one of those interchannels (grooves) considering the real triangular(valley)like geometry of the site. The DMC method solves stochastically the imaginary-time Schrödinger equation, ${ }^{18}$ and introduces, to reduce the variance of the energy obtained, what it is called a trial wave function. This function contains a summary of all the physical information of the system known a priori, and it usually has an analytic form with some parameters previously optimized by a variational Monte Carlo (VMC) calculation. The trial function for the $(10,10)$ interstice is

$$
\boldsymbol{\Psi}(\mathbf{R})=\prod_{i}^{3} \exp \left[-\frac{1}{2}\left(\frac{a}{r}\right)^{5}\right],
$$

where the $i$ index goes through the three CN's that surround the interstitial channel. $a$ was obtained from a VMC calculation for a single $\mathrm{Ne}$ atom inside the same setting and found to be $28.37 \AA$. This trial function is of the same type as that used for ${ }^{4} \mathrm{He}$ in a previous calculation. ${ }^{19}$ The groove wave function was taken to be of the same form and with the same parameters, except that the $i$ index went from 1 to 2, the number of tubes forming a groove. In both cases, $r$ is the distance of the $\mathrm{Ne}$ atom to the center of any of the tubes considered. The binding energies obtained by this method are $E_{b}^{\text {interst }}=1011.15(2) \mathrm{K}^{11}$ and $E_{b}^{\text {groove }}=659.48(2) \mathrm{K}$ at $0 \mathrm{~K}$, respectively. For both calculations the nanotubes have been
TABLE I. DMC calculations for the potential $\left(V_{b}\right)$, kinetic $\left(T_{b}\right)$, and total $\left(E_{b}\right)$ energies of a $(10,10)$ interstice, a $(10,10)$ groove, and a $(5,5)$ tube. $V_{\mathrm{Ne}-\mathrm{C}}$ is the value of the $\mathrm{Ne}-\mathrm{C}$ potential at the center of a $(10,10)$ interstice and a $(5,5)$ tube. All values expressed in $\mathrm{K}$.

\begin{tabular}{ccccc}
\hline \hline & $V_{\mathrm{Ne}-\mathrm{C}}$ & $V_{b}$ & $T_{b}$ & $E_{b}$ \\
\hline Interstice & -1096.18 & $-1062.75(2)$ & $51.60(2)$ & $1011.15(2)$ \\
Groove & & $-693.87(4)$ & $34.39(3)$ & $659.48(2)$ \\
Tube & -1477.64 & $-1410.25(4)$ & $2.1661(1)$ & $1408.08(4)$ \\
\hline \hline
\end{tabular}

considered to be infinite and smooth cylinders of radius $6.8 \AA$ and with a distance between centers of $17 \AA .{ }^{20}$ Different trial functions from those chosen would have given similar results for the binding energies, but with larger variances.

The $(5,5)$ tube has been modeled in a similar way: it was considered to be a long and featureless cylinder of $3.4 \AA$ of radius, with a distance between centers of cylinders of $10.2 \AA$. Its corresponding trial wave function was chosen as

$$
\boldsymbol{\Psi}(\mathbf{R})=\exp \left(-c r^{2}\right)
$$

where $c$ is another variational parameter, whose value was $0.765 \AA^{-2}$. This form of the trial was the same, with different parameters, as used in the case of ${ }^{4} \mathrm{He}$ (Ref. 21) and $\mathrm{H}_{2} .{ }^{22}$ According to these calculations, the binding energy of a neon atom inside a $(5,5)$ tube yields $E_{b}^{\text {tube }}=1408.08(3) \mathrm{K}$. In all cases, the parameters of the $\mathrm{Ne}-\mathrm{C}$ interaction were taken from Ref. 12.

Table I summarizes the DMC values of the potential, kinetic, and total binding energies obtained for each one of the systems described above, namely, the $(10,10)$ interstice, the groove of a $(10,10)$ bundle, and the $(5,5)$ tube. In that table we also give the value of the $\mathrm{Ne}-\mathrm{C}$ potential at the center of the $(10,10)$ interstice and the $(5,5)$ tube. Even though DMC is a zero-temperature technique, one can safely assume that the ground-state binding energy is going to be a good approximation to the energy of a single atom in the temperature range considered. This statement is based on the fact that potential energies obtained for each system are very similar to the corresponding value of the potential at the center of each channel.

Finally, the interactions between $\mathrm{Ne}$ atoms located in different interchannels or tubes are taken into account by a mean-field method, whose accuracy has been already checked in the case of $\mathrm{H}_{2} \cdot{ }^{23}$ The inclusion of this term is necessary, since it produces effects that could considerably affect the behavior of the quasi-one-dimensional gas inside the interchannel (for instance, liquid condensation ${ }^{11}$ ). The expression is

$$
E_{m}=\lambda \int_{0}^{\infty} d r V\left[\left(d^{2}+r^{2}\right)^{1 / 2}\right],
$$

where $V$ is the same $\mathrm{Ne}-\mathrm{Ne}$ potential used for atoms inside the same channel. $d$ is the minimum distance between the given channel and the one considered for the integration. $\lambda$ is the linear density of the $1 \mathrm{D} \mathrm{Ne}$ atoms in K/A. It is important to take into account all the surrounding interchannels neces- 
sary for the calculation to converge. To use only the three nearest neighbors conduces to the underestimation of this energy by at least $5 \%$. That integration yields for a bundle of $(10,10)$ tubes $-1.062 \lambda \mathrm{K}$, for neon atoms located on a groove $-0.687 \lambda \mathrm{K}$, and for a set of $(5,5)$ tubes $-1.645 \lambda \mathrm{K}$. This term was also taken to be independent of the temperature, but as in the case of the binding energy contribution, it is reasonable to consider it to be so. The total energy of neon atoms is then the sum of all three types of interactions: $\mathrm{C}-\mathrm{Ne}, \mathrm{Ne}$ - $\mathrm{Ne}$ in the same interstice or tube, and 1D Ne- $\mathrm{Ne}$ in different interstices or tubes.

From a typical PIMC calculation the parameter we get is the energy per atom, but what one needs to check the possibility of adsorption is the chemical potential, got by derivation from the free energy $F$. To estimate the free energy is possible if we have several energy isotherms. The strategy is to propose a reasonable form for $F$ as a function of temperature and density (its natural variables), and to deduce the corresponding expression for the energy by means of

$$
E(\lambda, T)=-T^{2} \frac{\partial[F(\lambda, T) / T]}{\partial T} .
$$

The expression chosen for the free energy per atom for a pure 1D system was ${ }^{24,25}$

$$
F(\lambda, T)=-k T \ln \left[\left(\frac{2 \pi m k T}{h^{2}}\right)^{1 / 2} \frac{e}{\lambda}\right]+\sum_{i=1}^{8} \sum_{j=1}^{3} a_{i j} \lambda^{i} T^{1-j},
$$

where $T$ is the absolute temperature, $m$ is the mass of a Ne atom, $e$ is the base of the natural logarithm, and $h$ is Plank's constant. The $a_{i j}$ 's indicate the different behavior of the system from that of an ideal 1D gas, represented by the first logarithmic term. ${ }^{26}$ The form of the equation assures that in the limits $T \rightarrow \infty$ and $\lambda \rightarrow 0$, we reproduce the energy and free energy of an ideal gas. This is the expression for a pure 1D gas without the $\mathrm{C}-\mathrm{Ne}$ interaction due to the carbon nanotubes and does not take into account the existence of $\mathrm{Ne}$ atoms in other interchannels or tubes. Equation (5) was devised to be used to obtain the free energy from PIMC calculations in a $2 \mathrm{D}$ system of ${ }^{4} \mathrm{He},{ }^{25}$ and was also successfully applied to obtain the free energy from classical molecular dynamics simulations of water, ${ }^{24}$ being a general expression that could be used for any type of calculation in a canonical ensemble whose output is the energy.

According to the previous form of the free energy, the energy per atom is

$$
E(\lambda, T)=\frac{k T}{2}+\sum_{i=1}^{8} \sum_{j=1}^{3} j a_{i j} \lambda^{i} T^{1-j} .
$$

Since we have four isotherms, we can make a least-squares fit of all the densities and all the temperatures together to this energy form. We will obtain then the $a_{i j}$ coefficients that will allow us to know the free energy from Eq. (6). Those coefficients are given in Table II. The $\chi^{2}$ per degree of freedom is less than 1 , which indicates that the quality of the model is excellent.
TABLE II. Values obtained for the parameters of the fit. $\chi^{2}$ per degree of freedom $=0.723043$.

\begin{tabular}{lrlrrr}
$a_{11}$ & $-7.7059 \times 10^{1}$ & $a_{12}$ & $3.73383 \times 10^{2}$ & $a_{13}$ & $-2.06986 \times 10^{4}$ \\
$a_{21}$ & $3.17676 \times 10^{3}$ & $a_{22}$ & $-6.99799 \times 10^{4}$ & $a_{23}$ & $1.06679 \times 10^{6}$ \\
$a_{31}$ & $-8.11551 \times 10^{4}$ & $a_{32}$ & $1.54315 \times 10^{6}$ & $a_{33}$ & $-2.33757 \times 10^{7}$ \\
$a_{41}$ & $9.49119 \times 10^{5}$ & $a_{42}$ & $-1.56391 \times 10^{7}$ & $a_{43}$ & $2.64569 \times 10^{8}$ \\
$a_{51}$ & $-6.12629 \times 10^{6}$ & $a_{52}$ & $8.66735 \times 10^{7}$ & $a_{53}$ & $-1.68287 \times 10^{9}$ \\
$a_{61}$ & $2.27492 \times 10^{7}$ & $a_{62}$ & $-2.78064 \times 10^{8}$ & $a_{63}$ & $6.12987 \times 10^{9}$ \\
$a_{71}$ & $-4.57469 \times 10^{7}$ & $a_{72}$ & $4.93201 \times 10^{8}$ & $a_{73}$ & $-1.19316 \times 10^{10}$ \\
$a_{81}$ & $3.86034 \times 10^{7}$ & $a_{82}$ & $-3.78165 \times 10^{8}$ & $a_{83}$ & $9.60314 \times 10^{9}$ \\
\hline \hline
\end{tabular}

From the free energy per atom, we can infer the 1D chemical potential by means of

$$
\mu(\lambda, T)=\frac{\partial(N F)}{\partial N},
$$

where $N$ is the number of atoms of the system, and $F$ is the free energy per atom. That gives

$$
\mu(\lambda, T)=-k T \ln \left[\left(\frac{2 \pi k m T}{h^{2}}\right)^{1 / 2} \frac{1}{\lambda}\right]+\sum_{i=1}^{8} \sum_{j=1}^{3} a_{i j}(i+1) \lambda^{i} T^{1-j},
$$

where, as before, the first term in the sum is the chemical potential of a purely 1D ideal gas. To go from this expression to that of a gas in an interstice, we have to sum up the corresponding binding energies and the $\mathrm{Ne}-\mathrm{Ne}$ interaction between $(10,10)$ interstices, or $(5,5)$ tubes (different for each case), given above, and add the correction due to the presence of other tubes $\left(2 E_{m}\right)$, i.e., $-2.124 \lambda$ in the first case, $-3.29 \lambda$ for a $(5,5)$ arrangement, and $-1.374 \lambda$ for $1 D$ neon adsorbed on a groove.

By summing up all the corresponding terms, we have already the free energy for a system of $\mathrm{Ne}$ atoms adsorbed inside the different locations considered. To check if adsorption is produced, we have to compare the chemical potential obtained with the one for the $3 \mathrm{D}$ gas outside the bundle. There, one can consider that neon is diluted enough to be described as a 3D ideal gas. Within this approximation, its chemical potential per atom is

$$
\mu(\rho, T)=-k T \ln \left[\left(\frac{2 \pi m k T}{h^{2}}\right)^{3 / 2} \frac{k T}{p}\right],
$$

in which we have used the well-known equivalence $p=\rho k T$. The reason is that the variable controlled in the experimental setups is the pressure, not the density of the outer gas. Matter trends to flow from regions of higher chemical potential to zones of lower chemical potential; therefore, if $\mu(\rho, T)$ $<\mu(\lambda, T)$ the gas would stay in the 3D phase, adsorbing in the interchannels or tubes in any other circumstances.

Until now, we have considered only one possibility of adsorption for neon atoms on the grooves: a one-dimensional set located in between two cylinders. However, grand canonical Monte Carlo calculations by Calbi and co-workers ${ }^{27}$ indicate that at low coverages there are two possible phases 
of Ne on top of grooves: a purely 1D one, and another in which three neon rows are disposed forming a triangularlike structure on top of the valleys between cylinders. We estimated also the chemical potential for this last phase for the sake of comparison, proceeding in a similar way to that described above instead of performing a full PIMC calculation for the new phase. To go from Eq. (8) above to the chemical potential of a gas in the triangular phase, we added, as in the 1D phase, the binding energy, and the term $-1.374 \lambda$ obtained from the interchannel correction. The binding energy was approximated as the average between the binding energy given before for an atom on the bottom of the valley, and the graphite binding energy for the other two atoms $\left(30 \mathrm{meV},{ }^{8}\right.$ experimental result). That value is $-451.92 \mathrm{~K}$. However, there is another term to add in this case, corresponding to the influence of the other $\mathrm{Ne}$ atoms on the groove on the chemical potential of a given one. We estimated that influence by an expression similar to Eq. (3):

$$
E_{\mathrm{Ne}-\mathrm{Ne}}=\frac{1}{3} \sum_{i=1}^{3} \sum_{j=i+1}^{3} \lambda \int_{-\infty}^{\infty} d x g_{1 \mathrm{D}}(r) V(r),
$$

with

$$
r=\left(x^{2}+y^{2}\right)^{1 / 2},
$$

$x$ and $y$ being the distances of an atom of reference row $i$ from one located on row $j$. To calculate those distances, we suppose that the perpendicular section of the phase is a perfect equilateral triangle, and the minimum distance between atoms is $3.09 \AA$ (the minimum of the $\mathrm{Ne}-\mathrm{Ne}$ interatomic potential). The equation above would be exact if the real correlation function for the triangular phase, $g(r)$, were considered. However, we do not have it, and introduced instead the one corresponding to a pure 1D phase in the same conditions of density and temperature. One expects this to be a better approach than to take a pure mean-field estimation, in which, as in Eq. (3), $g(r)$ would be 1 for all $r$ 's. Thus, to obtain the chemical potential of $\mathrm{Ne}$ in this triangular section, one would have to add up the 1D chemical potential given by Eq. (8), the average binding energy $\left[E_{b}^{\text {groove }}=659.48(2) \mathrm{K}\right]$, a term due to the Ne atoms adsorbed in the interstitial of the bundle of nanotubes $(-1.374 \lambda)$, and, finally, a contribution $2 E_{\mathrm{Ne}-\mathrm{Ne}}$, different for each temperature. This is so because the result of the integral in Eq. (10) is practically insensitive to $\lambda$ (apart, of course, from the $\lambda$ factor outside). The values of the integral for 40 and $50 \mathrm{~K}$ are $-163(2) \lambda$ and $-155(2) \lambda$, respectively. As above, an atom is supposed to stay in the $3 \mathrm{D}$ phase only if its chemical potential there is lower than that in the triangular groove phase.

\section{RESULTS}

The results of the fitting to obtain the $a_{i j}$ coefficients are given in Table II and displayed in Fig. 1. As mentioned before, the fitting procedure was carried out with the energy per atom. In this figure, the error bars are about the size of the symbols, and were not displayed for clarity. Those symbols represent the energies obtained using PIMC calculations. The lines are the result of the fit. The agreement is quite good, as

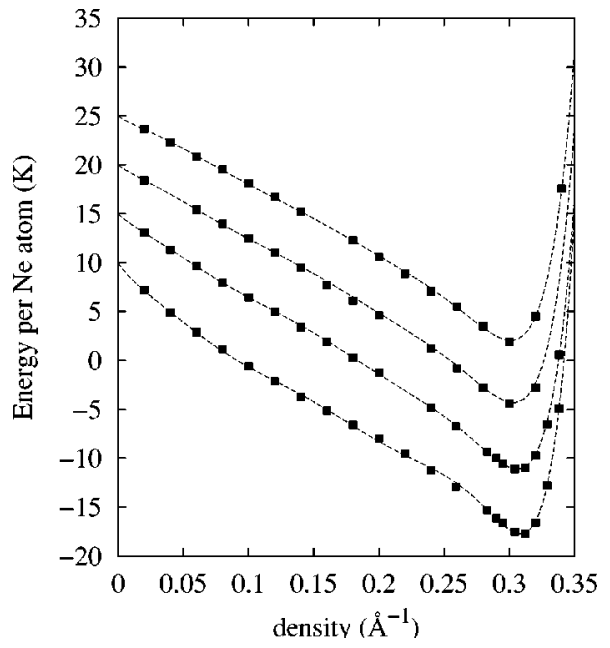

FIG. 1. Energy per neon atom as a function of density for a purely one-dimensional system. From top to bottom 50, 40, 30, and 20 K. Symbols correspond to PIMC simulations, lines correspond to fits.

is also deduced by the low value of the $\chi^{2}$ parameter per degree of freedom, and gives us some confidence in the chemical potentials obtained by this method. This procedure has also an additional advantage: with the fitting parameters, we can have the thermodynamical quantities we want at any intermediate temperature or density without doing any more calculations. We observe that for densities greater than $0.32 \AA^{-1}$ the energy increases heavily with density, a feature almost independent of the temperature. The reason for that behavior is that we have reached the limit in which neon atoms experience their neighbor's cores.

The chemical potentials $\mu$ for a pure 1D system deduced from the procedure described above are displayed in Fig. 2, where from top to bottom we have the results for 20, 30, 40, and $50 \mathrm{~K}$. Full lines are the results derived from simulations, while dashed lines are the same variables for a purely 1D

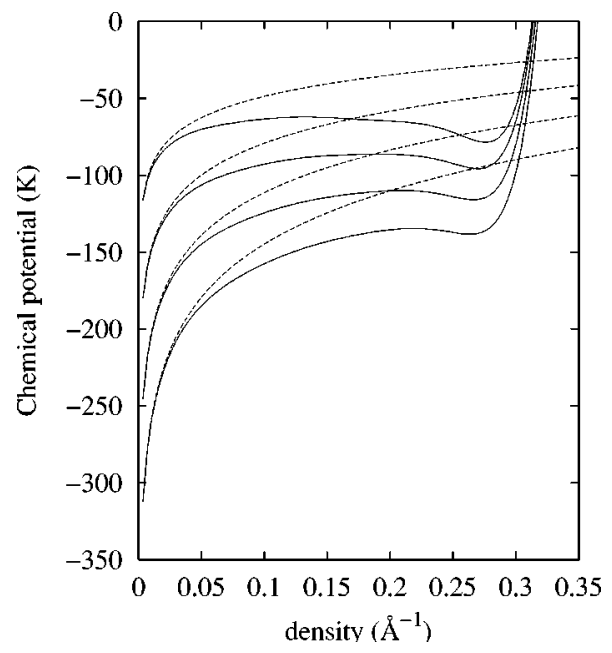

FIG. 2. Chemical potential for neon as a function of density for a purely one-dimensional system. From top to bottom 20, 30, 40, and $50 \mathrm{~K}$. Full lines correspond to the chemical potential deduced from Eq. (5), while dashed lines are the results for an 1D ideal gas. 


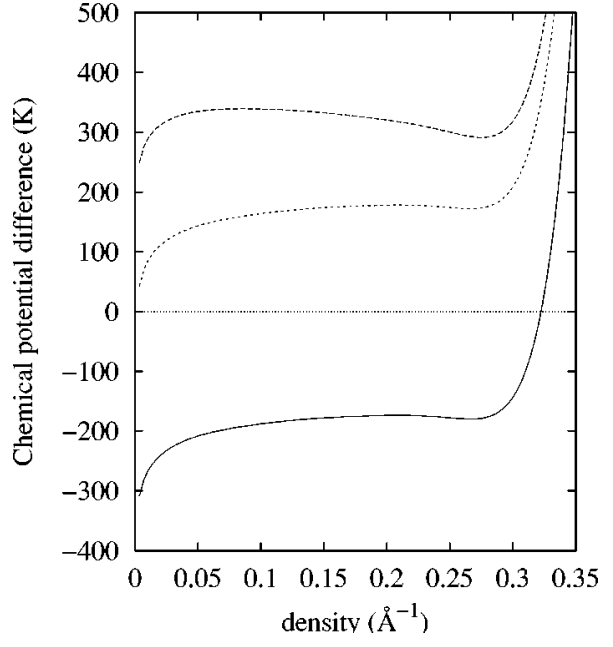

FIG. 3. Chemical potential difference between a neon atom inside an interchannel of a $(10,10) \mathrm{CN}$ bundle and neon atom in bulk (full line), neon in a 1D groove phase and neon in bulk (dotted lines), and neon in a triangular phase on top of a groove and neon in bulk (dashed line) at $40 \mathrm{~K}$. See further explanation in the text.

ideal gas (all $a_{i j}$ coefficients equal to zero). We can see that, as was to be expected, for very low densities, the system can be described accurately as a 1D ideal gas, and that the density range in which this is true increases with temperature.

Figure 3 shows the difference between the chemical potential of neon in the interstices and neon as a 3D ideal gas (full line), neon in a 1D phase on top of the grooves, and a 3D ideal gas (dotted line), and the same species on the triangular phase described above and the same 3D ideal neon (dashed line). If this difference is negative $\left(\mu-\mu_{3 \mathrm{D}}\right)$, adsorption occurs. It displays the data for $40 \mathrm{~K}$ and $p=9$ $\times 10^{-4}$ torr, a pressure that was chosen with the help of Ref. 8: we obtained it from extrapolation to zero adsorption on the grooves of the adsorption curve at $40.13 \mathrm{~K}$ in Fig. 1 of that reference. Our model predicts that the interstices are almost full of $\mathrm{Ne}\left(\Delta \mu<0\right.$ for $\left.\lambda \leqq 0.32 \AA^{-1}\right)$, while the grooves are clean, this last result in concordance with the experiment. We observe also an additional feature: at $40 \mathrm{~K}$ the favored phase on the grooves would be the 1D neon one, not the triangular phase between them.

In Fig. 4, we displayed the pressures at which the interchannels will begin to fill (lower curve) and be complete (upper curve) for different temperatures, ranging from 30 to $50 \mathrm{~K}$. The filling was arbitrarily defined to start when the $1 \mathrm{D}$ density in the tube is greater than $10^{-4} \AA^{-1}$, and we defined a tube as full when the same density is larger than $0.3 \AA^{-1}$. That means that above the upper curve all the interchannels are full, below the lower curve, the interstices are empty, and in between they are in the process of filling. That would mean than in some of the experimental conditions reported in Ref. 8, all the interstitials are full. To avoid the adsorption of $\mathrm{Ne}$ inside the interchannels at $40 \mathrm{~K}$ and pressures of the order of $10^{-6}$ torr, the binding energy should be about $700 \mathrm{~K}$, of the same order of magnitude as on the grooves, which seems not very reasonable.

Figure 5 is similar to Fig. 4 but for the case of a bundle of open $(5,5)$ tubes. As before, the lower curve is the start of

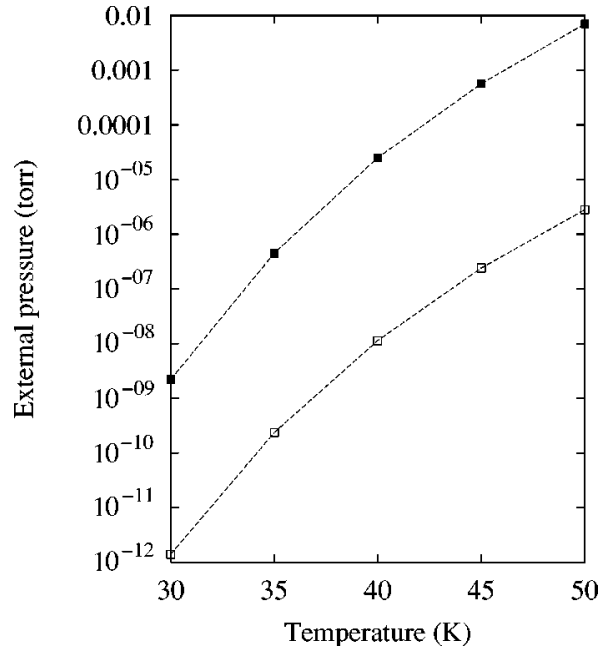

FIG. 4. Pressures at which the bundles start to fill (squares) and are full (full squares). The lines are guides to the eye.

filling $\left(\lambda>10^{-4} \AA^{-1}\right)$, the upper curve is the situation in which the tubes are full $\left(\lambda>0.3 \AA^{-1}\right)$, and the zone in between describes the process of filling. We can see also that the higher binding energy of $\mathrm{Ne}$ inside a $(5,5)$ tube makes it adsorb readily at much lower pressures than in the case of the $(10,10)$ interchannels.

Unfortunately, in the experimental data of adsorption of $\mathrm{Ne}$ on carbon nanotubes there is no indication of this species entering the interstices. When there is a discrepancy between the experimental and the theoretical results, the first suspect is the interatomic potential. To check its accuracy, we can compare the experimental binding energy of a $\mathrm{Ne}$ atom on top of a groove $(\sim 600 \mathrm{~K}$ in Ref. 8$)$ with what we obtain in our simulations: $E_{b}^{\text {groove }}=659.48(2) \mathrm{K}$, a value virtually identical to that of Ref. 12, $666 \mathrm{~K}$. Both numbers are within $10 \%$ of the experimental result, meaning that the Ne-tube potential used is reasonably good and should be adequate to study $\mathrm{Ne}$ in the slightly different environment of the interchannel tubes. Moreover, there is an independent estimation of this

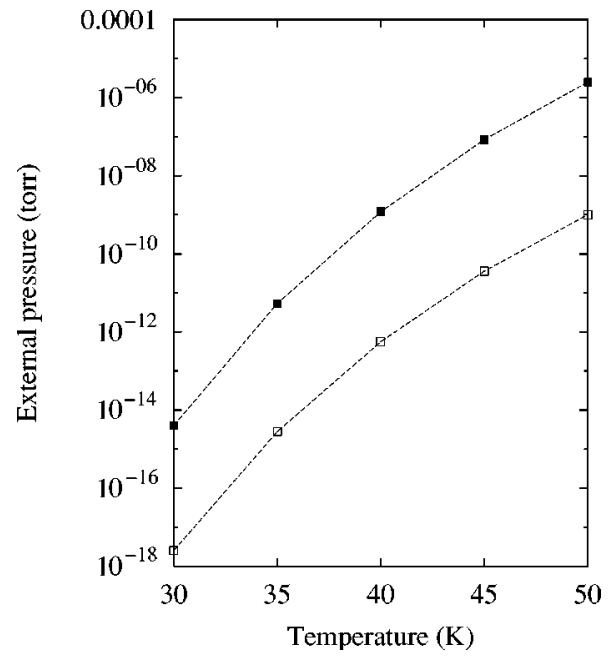

FIG. 5. Pressures at which the $(5,5)$ tubes in a bundle start to fill (squares) and are full (full squares). The lines are guides to the eye. 
one-particle energy in Ref. 12, done using a slightly different geometry of the interchannels (tubes of $6.9 \AA$ separated by $17 \AA$ instead of the $6.8 \AA$ radius we used), which gives a very similar value $\left(E_{b}=909 \mathrm{~K}\right)$ to what we presented in this work $\left(E_{b}=1011.15 \mathrm{~K}\right)$. That difference between the binding energies would not change the conclusions given here; neon should enter the interchannels but at slightly different external pressures. In that reference, there were also reported the energies of a single particle in the inside of the interchannels for other species: those of $\mathrm{Xe}$ and $\mathrm{CH}_{4}$ are positive. That would imply that, in agreement with the experimental results of Ref. 8, they do not enter the interstitial channels; simply they are too big. Obviously, the same energies of Xe and $\mathrm{CH}_{4}$ on the outside of the bundle are very negative, which means ready adsorption on the grooves.

However, the case of Ne should be different: the higher binding energy of $\mathrm{Ne}$ in the interchannels $(\sim 1000 \mathrm{~K})$ than in the grooves $(\sim 650 \mathrm{~K})$ should make it enter inside them unless there is some kind of entropic barrier to impede the process. This barrier comes from the fact that in a $3 \mathrm{D}$ environment the entropy of a gas is much greater than in a 1D location. The outcome of the experiment should be then a balance between the energy, which favors adsorption, and the entropy, which goes against it. We calculated then the difference of the chemical potentials between a $\mathrm{Ne}$ in a $3 \mathrm{D}$ environment and $\mathrm{Ne}$ in the interchannels, since this parameter takes into account the energy-entropy balance that governs adsorption. To our knowledge, this is the first time that the influence of entropy has been taken into account in a full PIMC calculation in this kind of system. The results, exemplified in Fig. 3, indicate that, at least in some circumstances, the entropy does not overcome the energy and we could observe adsorption inside the interchannels.

However, there are some other minor approximations to check before being reasonably sure of the correctness of our calculations and of the possibility of comparing them to the experimental results. First, we consider the carbon nanotubes here as featureless cylinders, while they really are the result of rolling a graphite sheet up to form a tube. That means that there is a certain corrugation that should be reflected in the $\mathrm{C}$-Ne potential and that has been neglected here. That effect has been considered by Boninsegni and co-workers ${ }^{28}$ in the ${ }^{4} \mathrm{He}$ case and found irrelevant both in the case of homogeneous interchannels, i.e., formed by three $(10,10)$ tubes, and for helium adsorbed in the inside of a single tube. However, it could be very important in the case of heterogeneous arrangements (two or three different tubes forming the interstices). The consequence is a localization of the atoms adsorbed in fixed places, which could clog the interchannels, and hinder the access of other atoms. We checked the influ- ence of corrugation in the Ne case in an interchannel formed by $(10,10)$ tubes perfectly aligned and with the carbon atoms in the same relative positions in each tube and found that the influence in the binding energy was less that $20 \mathrm{~K}$, which does not invalidate the results presented here. This is reasonable, since the corrugation length (the length of the unit cell of carbons) in a $(10,10)$ tube is $\sim 2.46 \AA$, smaller than the size of a neon atom. In addition, in an experimental situation one would expect the carbon atoms to be in different relative positions in each tube, which would decrease even further the influence of corrugation.

Obviously, another cause for the discrepancies between our results and the experiments is the experiments themselves. For instance, there is the question of the treatment given to the sample. In Ref. 8 it is claimed that the tubes are not prepared in any special way. To our knowledge, there is no other study of $\mathrm{Ne}$ in the interchannels of carbon nanotube bundles, but there are some experimental data for $\mathrm{H}_{2}$, a molecule slightly bigger than $\mathrm{Ne}^{4,5}$ These temperatureprogrammed-desorption experiments indicate that the bundles of carbon nanotubes have to be treated with $\mathrm{NaOH}$ to adsorb properly. This treatment disposes of any impurities in the system, especially hydrocarbons that could clog the interchannels and prevent the hydrogen entering to the adsorption sites. Again, there is no reason why there should not be a similar effect in the Ne case.

For all of the above reasons, we should expect our results to describe reasonably the adsorption of $\mathrm{Ne}$ in a clean unclogged bundle of homogeneous $(10,10)$ tubes. One possibility to check if the conclusions of this work are true is to carry out experiments in a setup in which, for a given temperature, we could have a small enough pressure to be under the line delimited by the open squares in Fig. 4, and then to increase the amount of gas let into the experimental cell. The idea is to monitor the amount of neon that disappears, if any, from the gas phase. However, the measurement should be done with extreme precision, due to the very low pressures involved (well below $10^{-6}$ torr).

Summarizing, we have performed a PIMC calculation that suggests that $\mathrm{Ne}$ atoms should enter the interstitial channels of $\mathrm{CN}$ bundles, at least for some experimental conditions. It will also adsorb easily in the inner part of a bundle of open $(5,5)$ tubes.

\section{ACKNOWLEDGMENTS}

The authors acknowledge A. D. Migone for insightful discussions. L.B. is grateful to C. Sbraccia for helpful advice on computational matters. This research was supported by the Grupo del Plan Andaluz de Investigación FQM-319 (Spain) and Italian Project No. MIUR-2001/025/498.
${ }^{1}$ J. Tersoff and R. S. Ruoff Phys. Rev. Lett. 73, 676 (1994).

${ }^{2}$ A. C. Dillon, K. M. Jones, T. A. Bekkedahl, C. H. Kiang, D. S. Bethune, and M. J. Heben, Nature (London) 386, 377 (1997).

${ }^{3}$ H. G. Schimmel, G. J. Kearley, M. G. Nijkamp, C. T. Visser, K. P. de Jong, and F. M. Mulder, Chem.-Eur. J. 9, 4764 (2003).
${ }^{4}$ M. Shiraishi, T. Takenoku, A. Yamada, M. Ata, and H. Kataura, Chem. Phys. Lett. 358, 213 (2002).

${ }^{5}$ M. Shiraishi, T. Takenoku, and M. Ata, Chem. Phys. Lett. 367, 633 (2003).

${ }^{6}$ M. M. Calbi, M. W. Cole, S. M. Gatica, M. J. Bojan, and G. Stan, 
Rev. Mod. Phys. 73, 857 (2001).

${ }^{7}$ W. Teizer, R. B. Hallock, E. Dujardin, and T. W. Ebbesen, Phys. Rev. Lett. 82, 5305 (1999); 84, 1844 (2000).

${ }^{8}$ S. Talapatra, A. Z. Zambano, S. E. Weber, and A. D. Migone, Phys. Rev. Lett. 85138 (2000).

${ }^{9}$ S. E. Weber, S. Talapatra, C. Journet, A. Zambano, and A. D. Migone, Phys. Rev. B 61, 13150 (2000); 66, 049901 (2002).

${ }^{10}$ S. Talapatra and A. D. Migone, Phys. Rev. Lett. 87, 206106 (2001).

${ }^{11}$ L. Brualla and M. C. Gordillo, Phys. Rev. B 68, 075423 (2003).

${ }^{12}$ G. Stan, M. J. Bojan, S. Curtarolo, S. M. Gatica, and M. W. Cole, Phys. Rev. B 622173 (2000).

${ }^{13}$ M. C. Gordillo, J. Boronat, and J. Casulleras, Phys. Rev. B 68, 125421 (2003).

${ }^{14}$ M. M. Calbi, F. Toigo, and M. W. Cole, Phys. Rev. Lett. 86, 5062 (2001).

${ }^{15}$ D. M. Ceperley, Rev. Mod. Phys. 67279 (1995).

${ }^{16}$ R. A. Aziz, and M. J. Slaman, Chem. Phys. 130, 187 (1989).

${ }^{17}$ C. Chakravarty, M. C. Gordillo, and D. M. Ceperley, J. Chem. Phys. 1092123 (1998).
${ }^{18}$ P. J. Reynolds, D. M. Ceperley, B. J. Alder, and W. A. Lester, J. Chem. Phys. 77, 5593 (1982).

${ }^{19}$ J. Boronat, M. C. Gordillo, and J. Casulleras, J. Low Temp. Phys. 126, 199 (2002).

${ }^{20}$ G. Stan and M. W. Cole, Surf. Sci. 395, 280 (1998).

${ }^{21}$ M. C. Gordillo, J. Boronat, and J. Casulleras, Phys. Rev. B 61, R878 (2000).

${ }^{22}$ M. C. Gordillo, J. Boronat, and J. Casulleras, Phys. Rev. Lett. 85, 2348 (2000).

${ }^{23}$ M. C. Gordillo, J. Boronat, and J. Casulleras, Phys. Rev. B 65, 014503 (2002).

${ }^{24}$ M. C. Gordillo and J. Martí, Phys. Rev. B 67, 205425 (2003).

${ }^{25}$ M. C. Gordillo and D. M. Ceperley, Phys. Rev. B 58, 6447 (1998).

${ }^{26}$ D. A. McQuarrie, Statistical Mechanics (University Science Books, Sausalito, CA, 2000).

${ }^{27}$ M. M. Calbi, S. M. Gatica, M. J. Bojan, and M. W. Cole, J. Chem. Phys. 115, 9975 (2001).

${ }^{28}$ M. Boninsegni, S. Y. Lee, and V. H. Crespi, Phys. Rev. Lett. 86, 3360 (2001). 